\title{
Sozialkapital als affektive Struktur sozialer Systeme
}

\author{
Paul Reinbacher*
}

Zusammenfassung: «Sozialkapital» ist in den letzten Jahren Gegenstand zahlreicher Untersuchungen gewesen, wobei man es allerdings meist formal als Netzwerkstruktur oder inhaltlich als Normenbestand definiert hat, um es letztlich zum modischen «umbrella term» für grundsätzlich bekannte soziologische Tatsachen zu machen. Demgegenüber wird hier vorgeschlagen, unter Rückgriff auf T. Parsons, N. Luhmann und H. Staubmann «soziales Kapital» über die affektive Dimension des Sozialen zu bestimmen.

Schlüsselwörter: Sozialkapital, Systemtheorie, Sinn, Affekt, Emotionen

\section{Le capital social comme structure affective des systèmes sociaux}

Résumé: Le "capital social» a fait l'objet de nombreuses études ces dernières années. D'un point de vue formel, il a le plus souvent été défini comme une structure de réseaux sociétaux et, d'un point de vue thématique, comme un ensemble de normes; il est par conséquent employé comme un terme générique englobant des faits sociologiques bien connus. Nous proposons, a contrario, de restreindre l'emploi du terme "capital social» à la dimension affective du monde social, suivant T. Parsons, N. Luhmann et H. Staubmann.

Mots-clés: capital social, théorie des systèmes, sens, affect, émotions

\section{Social Capital as Affective Structure of Social Systems}

Abstract: "Social capital" has been subject of numerous studies recently. However, it has mostly been defined formally as the structure of social networks or, with regard to its content, as a stock of social norms, which led to its usage as a fashionable "umbrella term" for basically well-known sociological facts. In contrast to this, we suggest reserving the term "social capital" primarily for the affective dimension of the social world, drawing from T. Parsons, N. Luhmann and H. Staubmann.

Keywords: social capital, systems theory, meaning, affect, emotions

\section{Einleitung: Sozialkapital als Modebegriff}

In der zeitgenössischen, zwischenzeitlich wieder abgeflauten Diskussion rund um jenes Kapital, das sich aus sozialen Beziehungen schlagen lässt (vgl. als Überblick z. B. Baron et al. 2000; Lesser 2000; Lin 2001; Lin et al. 2002; Halpern 2004), dominieren Zugriffe auf das Konzept Sozialkapital unter Rückgriff entweder auf formale Aspekte sozialer Strukturen (z. B. Netzwerkdichte) oder auf bestimmte Normenbestände (z. B. Reziprozität). In einigen Fällen findet sich überhaupt eine Gleichsetzung von

Pädagogische Hochschule Oberösterreich, AT-4020 Linz, paul.reinbacher@ph-ooe.at. 
Sozialkapital mit dem aus sozialen Zusammenhängen entstehenden Nutzen für Individuen und/oder Kollektive (z. B. ökonomisches Kapital, Wirtschaftswachstum etc.), womit Sozialkapital tautologisch über die Folgen von Sozialkapital bestimmt wird (vgl. z. B. kritisch Portes 1998). Abgesehen von so trivialen Feststellungen wie «Sozialkapital ist dort vorhanden, wo seine Folgen zu beobachten sind» stellt sich jedoch auch angesichts der soziometrischen Analysen von Netzwerken oder der soziologischen Untersuchung von Normen (vgl. z. B. Lüdemann und Peter 2007) die Frage nach der Zulänglichkeit dieser Perspektiven. Schliesslich ist es keineswegs unproblematisch, soziale Beziehungsgeflechte und ihre normative Dimension als Sozialkapital zu interpretieren, ohne mittels differentia specifica beispielsweise anzugeben, welche sozialen bzw. normativen Strukturen dann (noch) kein Sozialkapital sind, oder inwiefern negative Effekte dieser Strukturen die Nebenwirkungen von $z u$ viel Sozialkapital oder die Folgen von zu wenig Sozialkapital, also von social liability (Leenders und Gabbay 1999), sind (vgl. z. B. Woolcock und Narayan 2000; Franzen und Hangartner 2005; Weiss und Klein 2011).

Vor allem aber wäre zu klären, ob mit einem neuen umbrella term, der so unterschiedliche - und eigentlich bekannte - soziale Phänomene auf einen Nenner bringt, abgesehen von Aufmerksamkeit durch gekonntes Begriffsmarketing etwas gewonnen werden kann, oder ob der Begriff Sozialkapital nicht besser für eine bestimmte, beispielsweise wie hier vorgeschlagen für die affektive Dimension des Sozialen (den «expressiven Komplex» im Parsons'schen Sinne) zu reservieren wäre. Gerade dieser Aspekt kollektiver Emotionalität läuft in der Diskussion um Sozialkapital bislang eher latent mit, beispielsweise wenn von Vertrauen die Rede ist (vgl. z. B. Paxton 1999), obwohl bekanntlich neben Netzwerkstrukturen und Normen auch affektiv-emotionale Faktoren Bedeutung für den sozialen Zusammenhalt im Allgemeinen (vgl. bereits klassisch z. B. Durkheim 2003 [1897], 369; Durkheim 2005 [1912], 61-68; sowie ausführlich Gerhards 1988) und für soziales Kapital im Besonderen haben (vgl. z. B. Nahapiet und Ghoshal 1998; Paxton 1999; Putnam 2000; Schmid 2000; Adler und Kwon 2002).

Daher erscheint eine Reduktion des Sozialen oder des sozialen Kapitals auf eine Netzwerk- oder Normenstruktur problematisch. Unsere hier vorgeschlagene, über formale und normative Aspekte hinausgehende Beschreibung interpretiert demgegenüber Sozialkapital nach wie vor als emergentes, jedoch zuallererst expressivemotionales soziales Phänomen, das im Prozess affektiver Kommunikation produziert und reproduziert wird. Für uns ist Sozialkapital daher kein formales Beziehungsnetz unabhängig vom Inhalt (quasi die Infrastruktur des Sozialen), sondern in systemtheoretisch geprägter Perspektive eine Emergenz aus kommunikativen Operationen, die jedoch ebenfalls nicht vorschnell mit den Wissens- oder Normenbeständen eines Systems verwechselt werden sollte. Während wir also weiterhin die fundamentale Relevanz von Bedeutungsstrukturen (webs of significance nach C. Geertz 1973) für die «gesellschaftliche Konstruktion der Wirklichkeit» sensu P. Berger und Th. Luck- 
mann (2004 [1969]) anerkennen, wollen wir die apriorische Festlegung auf Sinn als das alleinige Substrat des Sozialen hinterfragen (vgl. im Anschluss an T. Parsons insbesondere Staubmann 1995; Staubmann 2004; Staubmann 2008). Folglich betrachten wir - wie auch in einem anonymen Gutachten gefordert ("Are affects and emotions not deeply interweaved with sense and meaning?") - Kognition und Emotion, Rationalität und Gefühl, Sinn und Affekt nicht nur auf individueller Ebene (vgl. z. B. Damasio 2004 [2000]; Damasio 2005 [1994]), sondern ebenso in der kollektiven Dimension (vgl. z. B. Ciompi 1997; Ciompi 2004; Ciompi und Endert 2011) als Korrelatbegriffe.

Kommunikation als Form des Sozialen (in der Tradition von z. B. G. Simmel, J. Derrida und insbesondere N. Luhmann) ist demnach nicht reduzierbar auf Operationen, die im Medium Sinn laufen (vgl. Luhmann 1984, Kap. 2), die sich also einer kollektiven (Sinn-)Semantik bedienen (vgl. Luhmann 1980b etc.) und die in weiterer Folge bloss auf individueller Ebene durch Gefühle als «Immunsystem» abgesichert werden (vgl. Luhmann 1984, 371). Vielmehr verarbeiten kommunikative soziale Akte auch Gefühle, indem sie für die Realisierung ihrer Formen das Medium Affekt verwenden (vgl. z. B. Parsons 1970 [1951], Kap. IX; Parsons 1974, 215). Als Bezeichnung für dieses emergente soziale Phänomen sui generis, für diesen expressiven Komplex (T. Parsons) bzw. für dieses web of affection zusätzlich zum Geertz'schen web of significance wird hier der Begriff Sozialkapital vorgeschlagen.

Die hinsichtlich Beobachtung und Beschreibung sozialer Wirklichkeit mit Universalitätsanspruch auftretende «Supertheorie» sozialer Systeme (vgl. z. B. Luhmann 1984, 19, 659) bietet sich mit ihrer formalen, differenztheoretischen Anlage für solche Überlegungen an, wenngleich einer ihrer blinden Flecken im Zuge von Beobachtungen zweiter Ordnung und mit Blick auf die Parsons'sche Vorgängertheorie in einer Derrida'schen Bewegung etwas verschoben werden muss: So tritt an die Stelle der üblichen Bestimmung des Sozialen über das Sinnhafte, also an die Stelle der Gleichsetzung von Sozialstruktur und Sinnstruktur eine doppelte Unterscheidung von Sinn vs. Affekt als Medien und individuell-psychische vs. kollektivsoziale Operationen als Formen, in denen beide Medien zum Ausdruck kommen. Wichtige Hinweise hierfür finden sich - wie von H. Staubmann gezeigt - bereits bei Klassikern wie T. Parsons, mit dem wir zwar (theoriegeschichtlich) zunächst einen Schritt zurück machen, dann jedoch über das Werk des sein Erbe antretenden N. Luhmann hinausgehen können; denn «[in] der Entwicklung der soziologischen Theorie nimmt das Werk Parsons' in vielerlei Hinsicht eine Schlüsselposition ein», wie nicht nur H. Staubmann $(1995,14)$ festgestellt hat, sondern auch N. Luhmann (1980a, 12) eingestehen musste:

Parsons ist deutlich über den ersten Schritt der Theorieentwicklung hinausgelangt. (...) Wer die Parsons'sche Reorganisation nicht akzeptiert, kann dennoch nicht auf den alten Stand zurück (...). Insofern ist dies Werk eine bleibende Herausforderung für alle weitere Arbeit an soziologischer Theorie. 
Nach einer kurzen Skizze verbreiteter Perspektiven auf Sozialkapital als Netzwerk und Normenbestand in Abschnitt 2 folgt daher hier ein Plädoyer für die Anerkennung der Mehrdimensionalität des Sozialen bzw. seiner Strukturierung in der Gestalt von Wissen, Normen und Sozialkapital in Abschnitt 3, bevor der Abschnitt 4 das empirisch zu untersuchende, nicht apriorisch zu behauptende Verhältnis dieser (zumindest drei) Aspekte diskutiert bzw. erste Hinweise auf solche empirische Evidenzen gibt und der Abschnitt 5 mit einem kurzen Fazit sowie mit kursorischen Hinweisen auf Limitationen und Implikationen die Überlegungen zu einem (vorläufigen) Abschluss bringt.

\section{Soziales Kapital als Netzwerk und Normenbestand}

Jene Diskussion um Bedeutung, Ursache und Nutzen von Sozialkapital, für die in den letzten zwei bis drei Jahrzehnten zunehmendes Interesse diagnostiziert werden kann, konzentriert sich trotz der grundsätzlich breiten Differenzierung der disziplinären Hintergründe und methodischen Herangehensweisen über weite Strecken auf die formalen Aspekte und die normative Dimension des Sozialen als Ursachen bzw. auf deren positive Wirkungen für Individuen und Kollektive. Kurz: "Social capital refers to the norms and networks that enable people to act collectively", wie M. Woolcock und D. Narayan $(2000,226)$ es im Sinne des Mainstreams zusammenfassen. Demgegenüber lässt sich allerdings vermuten, dass es gerade nicht nur formale Netzwerkstrukturen und sinnhafte Normen sind, die als Sozialkapital den Zusammenhalt und die Zusammenarbeit unterstützen, sondern dass dies eher über eine andere, nämlich die affektive Dimension mit expressiv-emotionalen Gratifikationen geleistet wird.

\subsection{Sozialkapital als Form: Netzwerk}

Ausgehend von prominenten Formulierungen wie jener P. Bourdieus $(1983,190)$, wonach Sozialkapital als "Gesamtheit der aktuellen und potentiellen Ressourcen, die mit dem Besitz eines dauerhaften Netzes von mehr oder weniger institutionalisierten Beziehungen gegenseitigen Kennens oder Anerkennens verbunden sind», zu verstehen ist, hat sich ein grosser Teil der Arbeiten zu diesem Thema ganz im Sinne eines Mehr-ist-besser-Denkens (z. B. Kontaktdichte, Vereinsmitgliedschaften o. ä.) auf die formalen Aspekte sozialer Zusammenhänge - die sogenannten sozialen Netzwerkstrukturen bzw. Beziehungsmuster - konzentriert (prominent z. B. Wasserman und Faust 1994). Damit wurde nicht zuletzt jene stark vereinfachende Vorstellung unterstützt, die das Sozial-Kommunikative insgesamt als Austausch materieller und immaterieller Ressourcenpakete auf der Basis interpersonaler Beziehungsnetze als Infrastruktur versteht (vgl. z. B. Blau 1964; Schulz von Thun 1981; Becker 1992). 
Unabhängig von der Betonung des Nutzens aus dichten oder lockeren sozialen Beziehungen - vgl. z. B. strong oder weak ties (Coleman 1988; Burt 1992; Burt 2002), bridging oder bonding capital (Putnam 1993) - sind es in diesem Verständnis Positionen in einem sozialen Gefüge inklusive der damit verbundenen Beziehungsund Kontaktmöglichkeiten, die als Ressourcen einzelnen Akteuren den Zugang zu weiteren Ressourcen eröffnen sollen. Dies haben seit P. Bourdieu (1983) vor allem die am Theoriestrang des Rational Choice orientierten, betont individualistischen Perspektiven herausgearbeitet (vgl. z. B. Baker 1990; Lin und Bian 1991; Burt 1992; Burt 1997a; Burt 1997b; Lin 1999; Burt 2000; Lin 2001; Burt 2002; Lin et al. 2002; auch Portes 1998 oder Meyerson 2000). Ein darüber hinausgehendes Verständnis von Sozialkapital als Eigentum bzw. Eigenschaft von Kollektiven oder als unbeabsichtigte Handlungsfolge und kollektives Nebenprodukt sozialen Handelns (vgl. z. B. Loury 1977 oder die populären Studien von Putnam 1993; Putnam 1995; Putnam 2000; Putnam 2001; Fukuyama 1995a; Fukuyama 1995b; zum Teil auch Coleman 1988; Coleman 1995) kann jedoch besser erklären, weshalb oft an der Hervorbringung Unbeteiligte von den positiven externen Effekten profitieren (vgl. z. B. auch Jansen 2002). Bereits für P. Bourdieu hatte sich der Begriff Sozialkapital schliesslich «angeboten zur Benennung des Prinzips der sozialen Wirkungen, von Wirkungen also, die zwar auf der Ebene der individuellen Handelnden (...) erfaßbar sind, ohne sich jedoch auf die Summe von individuellen Eigenschaften bestimmter Handelnder reduzieren zu lassen» (Bourdieu 1983, 191).

Insgesamt ist daher zunächst festzuhalten, dass, wie P. S. Adler und S.-W. Kwon (2002, 23-24) formulieren (vgl. ähnlich bereits Hanifan 1916),

[m] uch of research (...) locates the source of social capital in the formal structure of the ties that make up the social network (...) [and] has thus downplayed the importance of the contents of network ties (...) [which] implies that ties can have significant social capital benefits entirely independent of their content - an assumption we find implausible.

\subsection{Sozialkapital als Inhalt: Normen}

Die Durchsicht von Ansätzen, die Sozialkapital als soziale Netzwerkstruktur verstehen, fördert nämlich weisse Flecken zutage, die aus einer Beschränkung auf quantitativ messbare Kenngrössen, also z. B. aus der Kontaktdichte bzw. Kontakthäufigkeit zwischen Personen und anderen «Adressen» (vgl. Tacke 2000; Jansen 2002; Schechler 2002) resultieren, und eine zusätzliche Bestimmung auch des qualitativen Inhalts von Beziehungen - nicht nur als «Kontingenzvariable» (Burt 1992; Burt 1997a; Burt 1997b; Burt 2000) - erforderlich machen. Welche sozialen Verflechtungszusammenhänge wären sonst nämlich nicht per se Sozialkapital, oder zumindest potenzielles Sozialkapital, sofern (je nach Definition) beispielsweise eine bestimmte (messbare) Dichte der Beziehungen erreicht werden muss? 
R. D. Putnam, der hier als prominenter Vertreter stellvertretend für viele (vgl. z. B. Coleman 1988; Coleman 1995; Nahapiet und Ghoshal 1998; Paldam 2000; Paldam und Svendsen 2000; Woolcock und Narayan 2000) genannt werden kann, meint: "social capital refers to features of social organization, such as networks, norms, and trust, that facilitate coordination and cooperation for mutual benefit" (Putnam 1993, 35) bzw. "to connections among individuals - social networks and the norms of reciprocity and trustworthyness" (Putnam 2000, 19). Hier trifft er sich mit anderen Autoren wie beispielsweise mit F. Fukuyama (1995a; 1995b), der Sozialkapital über Vertrauen definiert und dieses ebenfalls als fundamental normenbasiert (based on shared norms) versteht. Doch so, wie soziales Kapital nicht mit den Strukturen sozialer Wirklichkeiten identisch ist, sollte man es auch nicht mit sozialen Normen gleichsetzen (vgl. z. B. Coleman 1988). Zumindest müsste - falls Sozialkapital nur eine Teilmenge der (ohnedies immer schon als solche beschreibbaren) Normen eines Sozialsystems darstellt - wenigstens ein Kriterium angegeben werden, das diesen Normenbereich von anderen Normen qualitativ-prinzipiell statt bloss enumerativ-graduell unterscheidbar macht, um ein neues Konzept wie Sozialkapital zu legitimieren.

Demgegenüber scheint allerdings die Attraktivität der Rede vom sozialen Kapital geradezu auf einer gewissen Unschärfe zu beruhen, denn "it may capture the essence of many sociological concepts (e.g., social support, social integration, social cohesion, and even norms and values) and serve as an umbrella term that can easily be understood and transported across many disciplines", wie Lin et al. (2002, vii) nicht ganz frei von Selbstkritik formulieren. In dasselbe Horn stossen auch P. S. Adler und S.-W. Kwon: Sie kritisieren dies als "bringing under the one notion much of what has been studied under such concepts as informal organization, trust, culture, social support, social exchange, social resources, embeddedness, relational contracts, social networks, and interfirm networks", wobei "[i]t is not obvious, however, that we gain more than we lose by gathering all these various phenomena under an 'umbrella concept' (...) of social capital" (Adler und Kwon 2002, 17-18). Demgegenüber liessen sich ja beispielweise schon mit M. Gluckman (1967) multiplex ties von simplex ties unterscheiden.

Insgesamt ist also festzuhalten, dass weder Versuche bloss formaler Annäherung an soziale Beziehungsgeflechte (vgl. Abschnitt 2.1) noch die Gleichsetzung mit generellen oder speziellen Normenbeständen (vgl. Abschnitt 2.2) eine soziologisch befriedigende Bestimmung von Sozialkapital erlauben.

\section{Soziale Strukturen als mehrdimensionale Emergenzen}

Die so gut wie allen Positionen der Debatte zugrunde liegende Verortung des - überwiegend positiv konnotierten - sozialen Kapitals bzw. seiner Grundlagen in sozialen 
Zusammenhängen lässt sich als Suche nach den positiven individuellen und kollektiven Effekten von Vergemeinschaftung und Vergesellschaftung ideengeschichtlich bis an die Anfänge der Sozialphilosophie bzw. der Soziologie zurückverfolgen. Denn dass "involvement and participation in groups can have positive consequences for the individual and the community is a staple notion dating back to Durkheim's emphasis on group life as an antidote to anomie", wie A. Portes $(1998,2)$ feststellt, wobei "research literature on social capital strongly emphasizes its positive consequences. Indeed it is our sociological bias to see good things emerge out of sociability; bad things are more commonly associated with the behavior of homo oeconomicus" eine Verzerrung, die sich gerade in der Diskussion über Sozialkapital hartnäckig hält.

Sofern man sich nicht mit Vorstellungen von sozialen Netzwerken als Infrastruktur zum Transport von Inhalten zufriedengibt, stellt sich die grundlegende Frage nach dem Stoff, aus dem solche sozialen Verflechtungszusammenhänge beschaffen sind, bzw. - systemtheoretisch formuliert - nach den Medien, mit denen die Formen zur Konstruktion der sozialen Wirklichkeit operieren.

Indem wir hier davon ausgehen, dass kommunikative Akte als Basisoperationen die Elemente bzw. die Fasern des Sozialen darstellen, und dass diese nicht Übertragungsleistungen, sondern komplexe Leistungen der Selektion bzw. der Synthese von Selektionen (wie Information, Mitteilung und Verstehen) sind, folgen wir einer formal angelegten Theorie sozialer Systeme, die von einer Kopplung analytisch unterscheidbarer Emergenzebenen - wie der psychisch-individuellen und der sozial-kollektiven - ausgeht (prominent: Luhmann 1984). Auf jeder dieser Ebenen sollen sich in einem laufenden Prozess Formen in Medien als Differenzen (J. Derrida) einschreiben. Psychisch-individuelle und sozial-kollektive Strukturen sind dementsprechend keine Gleiskörper, auf denen in Waggons die Ressourcen (Informationen, Wissen, Gefühle o. ä.) transportiert werden, sondern fortlaufend prozessierte Differenzen bzw. Formen-in-Medien, die als «Unterscheidungen, die einen Unterschied machen» (Bateson 1981 [1972], 576-597) ihre Spuren ziehen, sodass sich ein "Gewebe von Differenzen konstituiert» (Derrida 2004, 124; systemtheoretische Referenz ist in der Regel Spencer-Brown 1969).

Psychische und soziale Systeme gleichen sich demzufolge in ihrer Operationsweise der Differenzproduktion und unterscheiden sich nur durch die spezifische Form ihrer Basisoperationen (psychische Bewusstseins- vs. soziale Kommunikationsakte), in denen sie - davon sind wir im Anschluss an T. Parsons und H. Staubmann überzeugt - wiederum die gleichen Medien, nämlich Sinn (Bedeutung) und Affekt (Gefühl) verarbeiten, um letztlich sinnhafte und affektive Gewebe hervorzubringen: Wissens- und Normenstrukturen im Medium Sinn, Sozialkapitalstrukturen im Medium Affekt. 


\subsection{Wissen und Normen als sinnhafte Struktur}

In guter soziologischer und insbesondere systemtheoretischer Tradition gilt jedoch die genuin soziale Operation Kommunikation als ausschliesslich sinnhaft. Dies unterscheidet sie von den psychischen Operationen des Bewusstseins, denen neben sinnhaftem Denken auch affektiv-emotionales Fühlen zugestanden wird: Soziale Systeme konstituieren sich in diesem Verständnis durch die selektive Aktualisierung von Sinn unter Auswahl aus einem Horizont bestehender Möglichkeiten (vgl. z. B. Luhmann 1984, Kap. 2) - sind also ausschliesslich Sinn-Strukturen. Damit wird Sinn für die solchen verarbeitenden Systeme als unumgänglich und nicht hintergehbar gesehen - denn «Sinn verweist immer wieder auf Sinn und nie aus Sinnhaftem hinaus auf etwas anderes. Systeme, die an Sinn gebunden sind, können daher nicht sinnfrei erleben oder handeln» (Luhmann 1984, 96). Wissens- bzw. Normenbestände werden in weiterer Folge als sinnhafte Emergenzen zum Charakteristikum von Sozialsystemen interpretiert, indem sinnhafte Strukturen in Abhängigkeit von der (geplanten) Reaktion auf Umweltreize entweder als veränderbar und lernbereit oder - im Gegensatz dazu - als stur und enttäuschungsresistent klassifiziert werden (Luhmann 1969; Luhmann 1984, 396-399, 436-452). Die ersteren Strukturen bezeichnen wir üblicherweise als Wissen, die zweiteren als Normen. Darin (vgl. z. B. Luhmann 1984, Kap. 2) zeigt sich jedoch auf den zweiten Blick die cartesianischkognitivistische Verzerrung eines «intellektzentrierten Weltbildes» (Ciompi 1997, 11) auf Basis einer langen, vor allem westlichen wissenschaftlichen Tradition, die sich bis zu frühen, auch soziologischen Denkern zurückverfolgen lässt (vgl. Staubmann 1995; Staubmann 2004).

Man erinnere sich an die klassische Festlegung auf Handeln, mit dem die Handelnden einen «subjektiven Sinn» verbinden bei M. Weber (1980 [1922], \$1) oder an die weiteren Entwicklungslinien über E. Husserl, demzufolge die Welt ebenfalls stets sinnhaft ausgelegt und gedeutet werden muss, an A. Schütz (1932) mit seinem «sinnhaften Aufbau» der sozialen Welt bzw. an A. Schütz und Th. Luckmann $(1975,38)$ mit ihren «Strukturen der Lebenswelt», die sich uns als «subjektiver Sinnzusammenhang» präsentieren, bis hin zur "gesellschaftlichen Konstruktion der Wirklichkeit» bei P. L. Berger und Th. Luckmann (2004 [1969], 21), die meinen: «Die Alltagswelt breitet sich vor uns aus als Wirklichkeit, die von Menschen begriffen und gedeutet wird und ihnen subjektiv sinnhaft erscheint.»

Diesen paradigmatischen Entscheidungen folgend werden - wie in einer bereits wieder langsam verebbenden Publikationswelle jüngeren Datums durchwegs affirmativ konstatiert (vgl. z. B. Scherke 2009) - Affekte, Gefühle, Emotionen etc. als Aspekte der sozialen Wirklichkeit, d. h. als genuin soziale Phänomene weitgehend vernachlässigt. Es gibt in den Sozialwissenschaften keine prominente Theorie der Gefühle, meint D. Baecker $(2004,5)$ im Rahmen einer systemtheoretisch orientierten Auseinandersetzung namhafter Autoren (vgl. Soziale Systeme 10/1), womit er nahtlos bei N. Luhmann anschliesst, der in für ihn typischer Diktion trocken 
bemerkt hatte: «Die typische Aussage der Soziologie zu diesem Thema lässt sich recht einfach wiedergeben: Forschungslücke!» (Luhmann 1984, 370). Dieser Zustand wird meist umgehend plausibilisiert, denn: «Emotionen gelten als ein privates, persönliches und zugleich natürliches Phänomen» (Gerhards 1988, 12). Und so wird auf die Unmöglichkeit ihrer Behandlung als genuin soziale und damit soziologische Tatsachen hingewiesen, womit ihnen ein Platz in deren Reihen abgesprochen ist: «In der Tat entzieht sich der Tatbestand direkter soziologischer Behandlung. Die Soziologie könnte sich allenfalls mit der Kommunikation von Gefühlen (...) befassen» (Luhmann 1984, 370, Fussnote 39) - und er meint damit die sozialkollektive Kommunikation im sozialen Medium Sinn über individuell-psychisch im Medium Affekt erlebte Gefühle (vgl. z. B. Flam 2000; Illouz 2006; von Schewe 2009). Ungeachtet ihrer (unterschiedlich expliziten) Behandlung in den Arbeiten sogenannter Klassiker (vgl. Gerhards 1988; Scherke 2009), hat man Emotionalität als Phänomen- und Forschungsbereich im Rahmen der Ausdifferenzierung wissenschaftlicher Disziplinen der Individual- und Sozialpsychologie zugeschlagen, ohne eine Kommunikation mittels Gefühlen, das heisst: im Medium Affekt, ernsthaft zu überlegen. Dies aber versteht sich nicht von selbst! Gerade zeitgenössische systemtheoretische Perspektiven degradieren Gefühle (im Unterschied zu Vordenkern wie T. Parsons) zu rasch und apriorisch zu einem individuellen Immunsystem, dem vor allem beim Umgang mit enttäuschten Erwartungen als Funktion die Lösung interner Anpassungsprobleme als Abkühlungsfunktion zukommt (vgl. z. B. Luhmann z. B. 1984, 362-367; Baecker 2004; Ciompi 2004). Anhand dieser Fixierung auf Sinn als Inhalt des Sozialen diagnostiziert H. Staubmann (1995; 2004; 2008) sehr treffend einen blinden Fleck der Soziologie im Allgemeinen und der Systemtheorie im Besonderen. Der Grund für die Unfähigkeit, neben Sinn auch Affekt als soziales Phänomen zu sehen bzw. affektive Komponenten als integrale Bestandteile allen sozialen Geschehens anzuerkennen, sei vor allem ein «soziologischer Essenzialismus: Die Bindung des Sozialen an eine 〈Substanz»» (Staubmann 2004, 142), nämlich an Sinn als soziale Materie, was N. Luhmann in "eine Art phänomenologischen Reduktionismus» führt, obwohl doch "gerade mit dem Einbau des an sich abstrakten ¿inhaltsleeren`Systembegriffes die Lösung nahe gelegen wäre, die Frage der Inhalte sozialer Prozesse und Strukturen als empirische zu stellen und nicht durch apriorische Festlegungen zu präjudizieren» (Staubmann 2004, 146). Gerade die zeitgenössische Systemtheorie hätte ein Beobachtungsinstrumentarium zur Hand, um Gefühle bzw. Affekte auch als Inhalt der sozialen Form Kommunikation, nicht nur der individuellen Form Bewusstsein zu akzeptieren. Allerdings ergreift sie diese Gelegenheit einer zunächst nur formalen Bestimmung des System- bzw. Informations- und Kommunikationsbegriffs nicht, sondern alle Information - "a difference that makes a difference" (Bateson 1981 [1972], 582) - ist für sie stets sinnhafte Information, da für sie Kommunikation ausschliesslich im Medium Sinn vorstellbar ist. Demgegenüber ist jedoch zu vermuten, dass nicht nur sinnbasierte Kommunikation ein 
selektives Geschehen ist, das heisst, dass kommunikativ aktualisierte Selektionen nicht nur Bedeutungs- bzw. Sinn-Horizonte konstituieren können (Luhmann 1984, 194), sondern dass auch affektive Kommunikation selektive Wirkungen haben und Gefühls-Horizonte wie zum Beispiel «affektive Eigenwelten» (Ciompi 1997; Ciompi 2004) ausbilden kann. Menschen sind nicht nur in selbstgesponnene Bedeutungsgewebe, also in webs of significance (Geertz 1973) verstrickt, sondern ebenso über Gefühlsgewebe (webs of affection) mit anderen verbunden (vgl. Staubmann 1995, 93-144 sowie Reinbacher 2008).

\subsection{Sozialkapital als affektive Struktur}

Es lohnt sich also in Zusammenhang mit der Frage des sozialen Kapitals die Suche nach emergenten affektiven Strukturen als «Formen des Gedächtnisses in sozialen Systemen (...), die eher über das Präparieren von Emotionen laufen als über intellektuelle Kontrolle», wie D. Baecker $(2004,12)$ meint, und wie auch H. Staubmann (2004, 151-156) die Parsons'sche (vgl. z. B. Parsons 1970 [1951]; Parsons 1973 [1967]) Unterscheidung zwischen kognitiv-instrumenteller und affektiv-kathektischer Handlungsdimension in Erinnerung ruft. Die zweite dieser beiden Dimensionen ermöglicht demnach die Kommunikation von (also: mittels) Gefühlen in einem engeren Sinn und nicht nur jene über Gefühle, also das Prozessieren affektiver «gratifikatorischer Differenzen» (Staubmann 2004, 152). Systemtheoretisch formuliert wäre demnach konsequenterweise zu fordern, den «Informationsbegriff zunächst noch offen zu halten» (Staubmann 2004, 147). Denn wenn dieser ein Ereignis bezeichnet, das Systemzustände auswählt bzw. Strukturgebrauch aktualisiert und in weiterer Folge einen Struktureffekt hinterlässt (Luhmann 1984, 102), kann auch die Aktualisierung eines Affektes eine Selektion sein. Schliesslich verzichtet eine Vertrauensbeziehung auf Misstrauen, setzt sich Wohlwollen in angenehmer Weise von Missgunst ab und so weiter. Und wie auch Sinn stets auf weiteren Sinn vor dem Letzthorizont der Welt verweist, das heisst unnegierbar ist, so ist auch «das Dasein je schon immer gestimmt», wie L. Ciompi $(1997,69)$ an ein Diktum M. Heideggers erinnert.

Das hat bedeutende Konsequenzen für soziale Systeme bzw. für die Kommunikationen, aus denen sie bestehen. Denn wenn es für Sinn gilt, dass er entweder in der Form von Gedanken oder in der Form von Kommunikationen erscheint, so scheint es plausibel, anzunehmen, dass Gefühle zwar zunächst selbstverständlich als physisch-psychische Phänomene zu verstehen sind, jedoch nicht ausschliesslich solche bleiben müssen, da sie «eine andere Art der Irritation für soziale Systeme zur Verfügung stellen können als logisch-diskursive Gedanken bzw. ihre Kommunikation» (Simon 2004, 118-119), was auch die Interaktionsanalysen von R. F. Bales (1953) nahelegen: Wie Bedeutungen als Gedanken, das heisst als sinnhafte Differenzen eines psychischen Systems (Bewusstseins), nicht von aussen beobachtbar oder unmittelbar von einem anderen erschliessbar sind, müssen Gefühle bzw. affektive 
Differenzen ebenso erst kommunikativ in das soziale System eingespeist werden: «Wer sagt, was er denkt, entblößt sich genauso, wie der, der zeigt, was er fühlt», wie F. B. Simon $(2004,121)$ richtigerweise feststellt und wie auch L. Ciompi $(1997,249)$ Kommunikation als Form mit sowohl sinnhaften (kognitiven) als auch affektiven Inhalten interpretiert, denn «stark affektgesteuerte Komponenten (...) spielen in der verbalen wie nonverbalen Kommunikation eine mindestens gleich große Rolle wie die rein kognitiven Inhalte».

Bereits T. Parsons hat diese wechselseitige Nichtreduzierbarkeit betont, mit der «Konsequenz, dass Emotionen als soziale Phänomene eben nicht «zurückführbar» sind auf normative oder sinnhafte Regulierung», wie uns H. Staubmann $(2004,155)$ erinnert. Und er ist es auch, der damit die Relevanz des expressiven Komplexes als Ergänzung zum kognitiven Komplex betont (Staubmann 1995, 109-122, passim) und damit einen der zentralen Aspekte der stark empirisch-induktiv getriebenen Diskussion um Sozialkapital (vgl. z. B. Putnam 1993; Putnam 1995) theoretischdeduktiv vorwegnimmt:

[Bereits] Parsons versucht (...) zu zeigen, daß in Analogie zu diesem instrumentellen Aufbau der Gesellschaft in einem expressiven Komplex parallele Strukturen und Prozesse auf Grundlage der expressiven Handlungsdimension existieren. (...) Als paralleles Phänomen zur Kooperation in instrumentellen Beziehungen sieht Parsons einen expressiven Beziehungstypus, den er als Loyalität bezeichnet, und im Falle der Institutionalisierung als Solidarität. Institutionalisierung bedeutet bei Parsons, wie bereits ausgeführt, die normative Fixierung von Interaktionsmustern. Damit bedeutet Solidarität die normative Fixierung von Loyalität. (Staubmann 1995, 109-110)

Wie jedoch kollektive Wissens- und Normenbestände mehr als die Summe individueller kognitiver und normativer Vorstellungen sind (vgl. z. B. Luhmann 1969; Luhmann 1984, 444-452; Willke 1998), geht auch kollektive Emotionalität über die Summe bzw. über das synchrone Erleben von Individualgefühlen hinaus, sobald diese Gefühle affektiv kommuniziert werden, das heisst: nicht bloss sinnhaft über sie kommuniziert wird. Kommunikation als Form des Sozialen muss erst mit Affekten als Inhalt befüllt werden bzw. präziser: Sie muss erst Differenzen ins Medium Affekt einschreiben, um als soziale Operation neben sinnhaften auch affektive Selektionen anschlussfähig prozessieren zu können. Selbstverständlich ist kein (emergentes) soziales Phänomen in der Lage, unabhängig von den strukturell angekoppelten physischen und psychischen Systemen für sich zu existieren, denn kollektive Wissens-, Normen- und Sozialkapitalbestände müssen stets von Individuen mit-vollzogen werden, wenngleich sie diese transzendieren (vgl. z. B. Luhmann 1984, 142-143).

Weitaus interessanter als die Vorstellung einer wechselseitigen emotionalen Ansteckung von Individuen im Kollektiv (vgl. z. B. Ciompi 1997, Kap. 7; klassisch: Hatfield et al. 1992; Gump und Kulik 1997; früh auch bereits Collins 1981) schei- 
nen hier die genuin sozialen Emergenzen, das heisst: nicht nur quantitativ bemerkenswerte, sondern in erster Linie qualitativ neue Produkte rekursiver Vernetzung emotionaler Energien bzw. affektiver Kommunikationen (vgl. z. B. Ciompi 1997, 266-267, Kap. 7). Immerhin entsteht soziales Kapital aus den kommunikativen Operationen eines sozialen Systems, wobei es nicht nur um formale Vernetzung von Adressen (vgl. z. B. Tacke 2000), sondern um eine bestimmte inhaltliche Qualität geht, wie bereits von P. Bourdieu $(1983,190)$ bemerkt:

\section{Das Sozialkapital ist die Gesamtheit der aktuellen und potentiellen Ressour- cen, die mit dem Besitz eines dauerhaften Netzes von mehr oder weniger institutionalisierten Beziehungen gegenseitigen Kennens oder Anerkennens verbunden sind (...).}

Besser noch, er hätte den Hinweis auf die Notwendigkeit des Anerkennens hervorgehoben (vgl. auch Staubmann 1995, 86): Denn gegenseitiges Kennen im Sinne formaler Vernetzung von Adressen ist noch nicht ausreichend. Um von Sozialkapital reden zu können, bedarf es auch des Anerkennens und dementsprechend mehr als einer quantitativ messbaren Vernetzung in Beziehungsmustern. Es sind qualitative Aspekte erforderlich, die reine kollektive Dichte individueller Kontakte ist nicht genug. Beachtung erfordert vielmehr "the type of these connections, involving positive emotion" (Paxton 1999, 89-90) bzw. die Entwicklung und Pflege von "personal and emotional attachments" (Nahapiet und Ghoshal 1998, 244). Im Mittelpunkt steht jedenfalls "the quality of the set of relationships" (Szreter 2000, 57), denn erst der spezifische Gehalt von Kommunikation macht Sozialkapital möglich: Beziehungen sind "not interesting as mere 'contacts" (Putnam 2000, 20), wie P. Paxton (1999, 92-93) im Sinne P. Bourdieus klarer als andere herausstreicht, dass "social capital requires more than a network of ties, (...) network ties must also be of a particular type - trusting and positive"; und für A. A. Schmid (2000, 159): "social capital is a relationship of affinity and regard among people".

Darauf hat sich auch die oben geäusserte Vermutung bezogen, wonach sich mit T. Parsons erwarten lässt, dass es gerade nicht formale Netzwerkstrukturen und sinnhafte Normen (z. B. Reziprozität) sind, die Zusammenarbeit (z. B. Kooperation) in dieser Art und Weise ermöglichen bzw. erleichtern, sondern vielmehr die affektive Dimension des Sozialen (z. B. Loyalität) bzw. die damit verbundenen expressivemotionalen Gratifikationen. So formuliert nicht zuletzt H. Staubmann die These, «daß der Hauptfaktor der sozialen Integration in der Bindung an eine gemeinsame expressive Kultur liegt» (Staubmann 1995, 104). Das Konzept des sozialen Kapitals selbst sollte demzufolge nicht bloss auf die formalen und normativen Aspekte sozialer Beziehungsmuster reduziert werden, da es damit keine ausreichende Aussagekraft gewinnen kann: Soziale Beziehungen und kollektive Normen sind stets Ursachen individueller und kollektiver, erwünschter und unerwünschter Wirkungen - und 
damit keineswegs eine Entdeckung der Debatte über soziales Kapital, wie A. Portes (1998) richtig feststellt.

\section{Wurzeln von Sozialkapital und seine Wirkung auf Wissen bzw. Normen}

Unsere bisherigen Überlegungen machen die Notwendigkeit einer Unterscheidung von Entstehungs- und Wirkungszusammenhang im Fall von Sozialkapital unmittelbar deutlich (vgl. auch Portes 1998): Einerseits (vgl. Abschnitt 4.1) entsteht Sozialkapital als kommunikatives Phänomen aus der rekursiven Reproduktion affektiver Kommunikation, die sich in expressiver Sprache, Symbolik bzw. Semantik manifestiert (vgl. z. B. Langer 1963 [1942]; Langer 1976 [1953]; Foolen 1997; Gutzmann 2013). Andererseits (vgl. Abschnitt 4.2) lassen sich durch die Unterscheidung von Entstehungs- und Wirkungszusammenhang potenzielle positive bzw. negative Effekte dieser affektiven Struktur auf andere Dimensionen der Sozialstruktur, insbesondere auf die sinnhafte Kommunikation in Gestalt von Wissen und Normen identifizieren (vgl. z. B. Powell und Smith-Doerr 1994; Nahapiet und Ghoshal 1998; Adler und Kwon 2002; Reinbacher 2008; Andrews 2010). Dabei schlagen wir vor, diese Effekte als Kopplung strukturell unabhängiger Emergenzphänomene zu beschreiben, was bereits bei T. Parsons angelegt ist, da auch dieser expressiven und instrumentellen Komplex analytisch unterscheidet, wenngleich die beiden stets zwei Aspekte konkreter empirischer Interaktionen sind (explizit z. B. Staubmann 1995, 117-118).

\subsection{Wurzeln}

Sozialkapital- als affektive Systemstrukturen sind wie die sinnhaften Wissens- und Normenstrukturen im Kern eine Einschränkung der zwischen den Elementen des Systems zugelassenen Relationen. Dies bedeutet insofern eine Überführung unstrukturierter in strukturierte Komplexität, als der Grossteil möglicher Anschlussoperationen ausgeschaltet wird (Luhmann 1984, 383-384): «Diese Einschränkung konstituiert den Sinn von Handlungen", und wir ergänzen an dieser Stelle: sowie den affektiven Gehalt dieser Handlungen. Mit anderen Worten: Die kognitiven, normativen und affektiven Strukturen eines sozialen Systems sind interpretierbar als «Erwartungsstrukturen» (Luhmann 1984, 139-141), an denen sich festmachen lässt, womit vermutlich als nächstes zu rechnen ist, also: welche der möglichen kommunikativen Verknüpfungen wahrscheinlich sind und welche demgegenüber unwahrscheinlich - weil un-sinnig (im Fall von sinnhaften Strukturen wie z. B. Wissen und Normen) oder un-passend (im Fall von affektiven, wie z. B. ästhetischen Strukturen) - sind, weil «Morgen soll es regnen!» keine adäquate Antwort auf die Frage «Ist der Vertriebsleiter zu sprechen?» darstellt oder weil «ein gotischer Spitzbogen wohl kaum zwischen zwei Parthenonsäulen paßt, genausowenig, wie einige 
Swing-Takte inmitten «Nearer My God to Thee» fehl am Platz sind», wie T. Parsons (1986, 96-97) so treffend formuliert hat.

In empirischer Perspektive ist dabei die sinnhafte Dimension systemischer Erwartungsstrukturen wohl unmittelbar plausibel. Sie bezieht sich auf kommunikative Anschlussfähigkeit durch sinnvolle Beiträge, wie zum Beispiel: «Ist der Vertriebsleiter zu sprechen?» - «Worum geht es?» - «Ich hätte eine Rückmeldung: Bei den letzten Lieferungen gab es ungewöhnliche Verzögerungen.» - «Einen Moment, bitte, ich verbinde!» usw., also um das, was A. Foolen (1997) als inhaltliche Funktion der Sprache bezeichnet hat. Die affektive Dimension derartiger systemischer Erwartungsstrukturen findet weit weniger Beachtung, doch lässt sie sich über die Foolen'sche expressive Funktion identifizieren: So könnte der Hinweis einer Kundin am Telefon auf jüngste Lieferprobleme abgesehen vom Versuch, den Vertriebsleiter zu erreichen, vorher noch die Reaktion «Ich entschuldige mich für die Unannehmlichkeiten!» auslösen, was einer expressiv neutral gekleideten Entschuldigung entspricht. Demgegenüber wären «Oh, das bedauern wir!» oder gar «Oh, das tut uns leid!» zwar inhaltlich vergleichbare, weil ebenfalls einer Entschuldigung entsprechende, jedoch deutlich expressivere Formulierungen, die zudem in positiver Weise auf die Formulierung der Anruferin reagieren, also auch affektive Anschlussfähigkeit sicherstellen: «Das kann ich mir nicht vorstellen (dass wir einen Fehler gemacht haben), aber wenn es sein muss, verbinde ich sie eben ...!» würde die Kommunikation zwar in der sinnhaften Dimension tragen, doch in der affektiven wohl kaum - wie der gotische Spitzbogen die Parthenonsäulen.

Bereits die Interaktionsanalysen von R. F. Bales (1953, hier 122) haben gezeigt, dass "when the last act was one of tension release, the next act is likely to be another another act of tension release". Oder umgekehrt mit L. Ciompi $(2004,38)$ : «Auf eine ärgerliche Kommunikation folgt viel wahrscheinlicher eine ebenfalls ärgerliche als eine gegenteilige Reaktion.» Von Bedeutung ist hierbei jedoch vor allem, dass es bei expressiver Kommunikation nicht darum geht, durch sinnhafte soziale Operationen Informationen über affektive psychische Zustände zu vermitteln (so z. B. Luhmann 1982; kritisch dazu z. B. Staubmann 1995, 100-102), sondern genuin soziale Affekte mit expressiven bzw. präsentationalen Symbolen (Parsons 1970 [1951], Kap. IX; Langer 1963 [1942]; Langer 1976 [1953]) zu verarbeiten. So funktioniert, wie uns H. Staubmann (1995, 232-245) erinnert, neben dem symbolisch generalisierten Medium Einfluss (Parsons 1963) auch Prestige (Veblen 1973): Als expressive/präsentationale Symbole können die beiden zwar sinnhaft/diskursiv interpretiert werden (vgl. Bourdieu 2003 [1979]) - sie müssen dies jedoch nicht und können trotzdem das soziale Handeln von Akteuren integrieren, indem sie doppelte Kontingenz dadurch überwinden helfen, dass Alter durch sie motiviert wird, die Situationsdefinition von Ego zu übernehmen. Diese «expressive Führungsrolle» (Parsons 1970 [1951], 399-407) entspricht dabei jener individuellen Ausstattung mit sozialem Kapital, die den Zugang zu ökonomischem, kulturellem oder symbolischem Kapital erleichtert. 
Für unsere Analyse ist jedoch in erster Linie bedeutsam, dass diese Wirkung dabei auf einer Hintergrundstruktur beruht, die nicht auf Sinn reduzierbar ist.

\subsection{Wirkungen}

Bezogen auf das Verhältnis zwischen affektiven und sinnhaften Strukturen zeigen beispielsweise einerseits Berichte aus der Kulturanthropologie, "dass die sich am langsamsten verändernden Regeln von Kulturen [also: normative sinnhafte Strukturen] durch affektive Kommunikation erlernt und weitergegeben werden» (Simon 2004, 127) - in der hier gewählten Terminologie: dass rekursiv vernetzte sinnhafte Kommunikationen durch die Engführung mit affektiven gefestigt, aber dennoch weiterhin als je eigene Wirklichkeitsdimension reproduziert werden und beobachtbar bleiben. Andererseits wird (als gewissermassen gegenläufiger Effekt) in der Diskussion um Sozialkapital die Bedeutung von Normen, also von vorrangig sinnhaften Strukturen (z. B. Reziprozität) als Entstehungsbedingung für affektive Emergenzen betont: Affektive Kommunikation findet im normativ gesicherten sozialen Raum bessere Entstehungsbedingungen vor. Eine Unterscheidung ist allerdings erforderlich, denn eine Gleichsetzung von (sinnhafter) Norm- und (affektiver) Sozialkapitalstruktur, wie seit frühen Arbeiten verbreitet anzutreffen, kommt einem Kategorienfehler gleich (vgl. z. B. Putnam 1993; Putnam 1994).

Auch L. Ciompi (vgl. z. B. 1997; 2004, 38-41) spricht im Rahmen seiner Affektlogik von affektiven Schienen zwischen Individuen, die Kommunikation wahrscheinlicher machen, indem jeder angeschlossene Durchgang die bereits gebahnten Wege vertieft und so emotionale Energie spart. Dies betrifft letztlich auch das Verhältnis von affektiven zu kognitiven sinnhaften Strukturen, also: Wissen - man denke zum Beispiel an Wissens-Communities (früher: Gilden, heute: Communities of Practice), in die von der Managementlehre grosse Hoffnungen in Bezug auf individuelle und kollektive Lernprozesse gesetzt werden (vgl. z. B. Brown und Duguid 1991; Wenger et al. 2002). In der Tat kann affektive Kommunikation die kollektive Identität absichern und den kognitiven Strukturen Flexibilität für Veränderung (= Lernen) verschaffen. Doch ist dies nicht zwingend der Fall, denn mitunter werden kollektive Wissensbestände emotional imprägniert bzw. durch affektive Attraktoren in einer Art und Weise abgelenkt und stabilisiert, die deren Lernbereitschaft beeinträchtigt und das soziale System in einen Ort sturen Beharrens (= Nichtlernen) verwandelt (vgl. z. B. Janis 1972; Wenger et al. 2002; Reinbacher 2008). Ähnliches haben Nahapiet und Ghoshal (1998) festgestellt, jedoch ohne die sinnhafte Wissens- und die affektive Sozialkapitaldimension der Kommunikation in der Analyse zu unterscheiden, sodass mit dem modischen umbrella term verwischt wird, was sich analytisch zu differenzieren lohnt. 


\section{$5 \quad$ Fazit, Limitationen und Implikationen}

Sozialkapital sollte den hier präsentierten Überlegungen zufolge also weder formal mit Netzwerken als Beziehungsgeflechten gleichgesetzt, noch inhaltlich auf bestimmte Normen der Sozialstruktur reduziert, oder überhaupt tautologisch über die positiven Effekte sozialer Verflechtungszusammenhänge definiert werden, da dies - abgesehen von der neuen Begrifflichkeit - keine hinreichende qualitative Unterscheidung des Konzepts von ohnehin bekannten soziologischen Tatsachen ermöglicht. Demgegenüber wurde hier eine innovative konzeptuelle Bestimmung von Sozialkapital ausgehend von der oft übersehenen Mehrdimensionalität der sozialen Wirklichkeit versucht. Diese stellt sich nämlich für die Soziologie üblicherweise und in erster Linie als kommunikative Konstruktion im Medium Sinn dar. Allerdings hat sie darüber hinaus auch eine fundamental affektive Dimension. Diese im Mainstream soziologischer Debatten gern vernachlässigte, genuin soziale affektive Emergenz entspricht dabei über weite Strecken sowohl dem, was T. Parsons einst den expressiven Komplex genannt hat, als auch dem, was die zeitgenössische Diskussion als Sozialkapital bezeichnet. Davon ausgehend lässt sich dann deduktiv nach potenziellen, sowohl positiven als auch negativen Wirkungen auf die sinnhafte Dimension des Sozialen - wie Wissens- und Normenbestände - suchen.

Der hier gewählte, auf analytische Differenzierung und synthetische Integration gegründete Zugang unterscheidet sich damit - und darauf wurde nicht zuletzt in einem anonymen Gutachten hingewiesen - grundsätzlich von bisherigen Studien über soziales Kapital, wo Normen (z. B. Kooperation und Reziprozität), Wissen (z. B. Kennen und Anerkennen) und Affekt (z. B. Vertrauen und Loyalität) vermengt werden. Möglicherweise wäre es zwar in der Tat akademisch anschlussfähiger bzw. «strategisch klüger» (wie ein Gutachter bzw. eine Gutachterin es formuliert), an diese etablierte Begriffsverwendung anzuschliessen und Sozialkapital nicht auf eine affektive Emergenz zu reduzieren. Dies allerdings hiesse: einerseits eine neue Begrifflichkeit für ebendiese affektive Emergenz suchen zu müssen (wie z. B. emotionales Kapital) oder zumindest existierende Konzepte neu zu interpretieren (wie z. B. die Durkheim'schen «Kollektivgefühle» oder den Parsons'schen «expressiven Komplex»), um sie für zeitgenössische Theorien wie beispielsweise die Luhmann'sche brauchbar zu machen, sowie andererseits die Suche nach jenem qualitativen Kriterium, das Sozialkapital von anderen Normen-, Wissens- und Affektstrukturen unterscheidet, von vorn zu beginnen (vgl. die Einleitung oben).

In jedem Fall ergeben sich aus den hier angestellten Überlegungen (neue) Herausforderungen für die empirische Sozial(kapital)forschung, da diese - insbesondere in ihrer (ohnedies seltenen) qualitativen Ausrichtung - nun gefordert ist, zusätzlich zur Rekonstruktion von Sinnstrukturen durch jene «besondere Form der Sinnverarbeitung (...), die man “Verstehen` nennt» (Luhmann 1984, 101), auch Mittel und Wege zu finden, um Affektstrukturen methodologisch in den Griff zu 
bekommen und mit geeigneten Methoden einen Zugriff auf sie zu erlangen (vgl. die kursorischen Hinweise oben in Abschnitt 4). Man denke beispielsweise nur an die auf G. Ryle zurückgehende dichte Beschreibung zur Rekonstruktion der Bedeutungsgewebe (Geertz 1973): Durch ihr Operieren mit diskursiven Symbolen (Langer 1963 [1942]; Langer 1976 [1953]) stösst sie an Grenzen, wenn es um die Analyse von Affektgeweben geht, denn diese legen die Rekonstruktion über einen expressiven bzw. "präsentationalen» Symbolismus - und mithin eine künstlerischästhetische Forschungsperspektive (vgl. z. B. Mersch und Ott 2007; Tröndle und Warmers 2012; Badura et al. 2015) - nahe, ohne dass im Gegenzug auf die diskursive Anschlussfähigkeit im System wissenschaftlicher Kommunikation verzichtet werden darf. Somit lässt sich abschliessend noch die These formulieren, dass die bis dato nur mangelhafte Auseinandersetzung mit der affektiven Dimension des Sozialen nicht nur, aber ganz besonders auch in Studien zu Sozialkapital nicht zuletzt auf die methodologischen und methodischen Schwierigkeiten ihrer (empirischen) Aufschliessung zurückzuführen sind, sodass hier ein wesentlicher Ansatzpunkt für Anschlussarbeiten liegt.

\section{$6 \quad$ Literatur}

Adler, Paul S. und Seok-Woo Kwon. 2002. Social capital. Prospects for a new concept. Academy of Management Review 27(1): 17-40.

Andrews, Rhys. 2010. Organizational social capital, structure and performance. Human Relations 63(5): 583-608.

Badura, Jens, Selma Dubach, Anke Haarmann, Dieter Mersch, Anton Rey, Christoph Schenker und Germán Toro Pérez (Hrsg.). 2015. Künstlerische Forschung. Ein Handbuch. Zürich: diaphanes.

Baecker, Dirk. 2004. Einleitung: Wozu Gefühle? Soziale Systeme 10(1): 5-20.

Baker, Wayne E. 1990. Market networks and corporate behavior. American Journal of Sociology 96(3): 589-625.

Bales, Robert F. 1953. The equilibrium problem in small groups. S. 111-162 in Working Papers in the Theory of Action, hrsg. von Talcott Parsons, Robert F. Bales und Edward A. Shils. New York: Free Press.

Baron, Stephen, John Field und Tom Schuller (Hrsg.). 2000. Social Capital. Critical Perspectives. Oxford: Oxford University Press.

Bateson, Gregory. 1981 [1972]. Ökologie des Geistes: Anthropologische, psychologische, biologische und epistemologische Perspektiven. Frankfurt a. M.: Suhrkamp.

Becker, Gary S. 1992. The economic way of looking at life. Nobel Lecture, December 9, 1992.

Berger, Peter L. und Thomas Luckmann. 2004 [1969]. Die gesellschaftliche Konstruktion der Wirklichkeit. Frankfurt a. M.: Fischer.

Blau, Peter M. 1964. Exchange and Power in Social Life. New York: Wiley.

Bourdieu, Pierre. 1983. Ökonomisches Kapital, kulturelles Kapital, soziales Kapital. Soziale Welt Sonderband 2: 183-198.

Bourdieu, Pierre. 2003 [1979]. Die feinen Unterschiede. Kritik der gesellschaftlichen Urteilskraft. Frankfurt a. M.: Suhrkamp. 
Brown, John S. und Paul Duguid. 1991. Organizational learning and communities-of-practice. Organization Science 2(1): 40-57.

Burt, Ronald S. 1992. Structural Holes. The Social Structure of Competition. Cambridge: Harvard University Press.

Burt, Ronald S. 1997a. The contingent value of social capital. Administrative Science Quarterly 42(2): 339-366.

Burt, Ronald S. 1997b. A note on social capital and network content. Social Networks 19(4): 355-373.

Burt, Ronald S. 2000. The network structure of social capital. Research in Organizational Behavior 22(2): 345-423.

Burt, Ronald S. 2002. Structural holes versus network closure. S. 31-56 in Social Capital. Theory and Research, hrsg. von Nan Lin, Karen Cook und Ronald S. Burt. New York: De Gruyter.

Ciompi, Luc. 1997. Die emotionalen Grundlagen des Denkens. Entwurf einer fraktalen Affektlogik. Göttingen: Vandenhoeck \& Ruprecht.

Ciompi, Luc. 2004. Ein blinder Fleck bei Niklas Luhmann? Soziale Wirkungen von Emotionen aus Sicht der fraktalen Affektlogik. Soziale Systeme 10(1): 21-49.

Ciompi, Luc und Elke Endert. 2011. Gefühle machen Geschichte. Die Wirkung kollektiver Emotionen - von Hitler bis Obama. Göttingen: Vandenhoeck \& Ruprecht.

Coleman, James S. 1988. Social capital in the creation of human capital. American Journal of Sociology 94 (Supplement): S95-S120.

Coleman, James S. 1995. Grundlagen der Sozialtheorie. Band 1. Handlungen und Handlungssysteme. München: Oldenbourg.

Collins, Randall. 1981. The microfoundations of macrosociology. American Journal of Sociology 86(5): 984-1014.

Damasio, Antonio R. 2004 [2000]. Ich fühle, also bin ich. München: List.

Damasio, Antonio R. 2005 [1994]. Descartes' Irrtum. Fühlen, Denken und das menschliche Gehirn. Berlin: List.

Derrida, Jacques. 2004. Die différance. Ausgewählte Texte. Stuttgart: Reclam.

Durkheim, Émile. 2003 [1897]. Der Selbstmord. Frankfurt a. M.: Suhrkamp.

Durkheim, Émile. 2005 [1912]. Die elementaren Formen des religiösen Lebens. Frankfurt a. M.: Suhrkamp.

Flam, Helena. 2000. The Emotional "Man" and the Problem of Collective Action. Frankfurt a. M.: Lang.

Foolen, Ad. 1997. The expressive function of language. S. 15-31 in The Language of Emotions. Conceptualization, Expression, and Theoretical Foundation, hrsg. von Susanne Niemeier und René Dirven. Amsterdam, Philadelphia: John Benjamins.

Franzen, Axel und Dominik Hangartner. 2005. Soziale Netzwerke und beruflicher Erfolg. Eine Analyse des Arbeitsmarkteintritts von Hochschulabsolventen. Kölner Zeitschrift für Soziologie und Sozialpsychologie 57(3): 443-465.

Fukuyama, Francis. 1995a. Social capital and the global economy. Foreign Affairs 74(5): 89-103.

Fukuyama, Francis. 1995b. Trust. The Social Virtues and the Creation of Prosperity. New York: Free Press.

Geertz, Clifford. 1973. Thick description. Toward an interpretative theory of culture. S. 3-30 in The Interpretation of Cultures. Selected Essays, hrsg. von Clifford Geertz. New York: Basic Books.

Gerhards, Jürgen. 1988. Soziologie der Emotionen. Fragestellungen, Systematik und Perspektiven. Weinheim: Juventa.

Gluckman, Max. 1967. The Judicial Process Among the Barotse of Northern Rhodesia. Manchester: Manchester University Press.

Gump, Brooks B. und James A. Kulik. 1997. Stress, affiliation, and emotional contagion. Journal of Personality and Social Psychology 72(2): 305-319. 
Gutzmann, Daniel. 2013. Expressives and beyond. An introduction to varieties of use-conditional meaning. S. 1-58 in Beyond Expressives. Explorations in Use-conditional Meaning, hrsg. von Daniel Gutzmann und Hans-Martin Gärtner. Leiden: Brill.

Halpern, David. 2004. Social Capital. Oxford: Polity.

Hanifan, Lyda J. 1916. The rural school community centre. Annals of the American Academy of Political and Social Sciences 67: 130-38.

Hatfield, Elaine, John T. Cacioppo und Richard L. Rapson. 1992. Primitive emotional contagion. Review of Personal and Social Psychology 14(1): 151-177.

Illouz, Eva. 2006. Gefühle in Zeiten des Kapitalismus. Frankfurt a. M.: Suhrkamp.

Janis, Irving L. 1972. Victims of Groupthink. A Psychological Study of Foreign-policy Decisions and Fiascoes. Boston: Houghton Mifflin.

Jansen, Dorothea. 2002. Netzwerkansätze in der Organisationsforschung. Kölner Zeitschrift für Soziologie und Sozialpsychologie Sonderheft 42: 88-118.

Langer, Susanne K. 1963 [1942]. Philosophy in a New Key. Cambridge: Harvard Univ. Press.

Langer, Susanne K. 1976 [1953]. Feeling and Form. London: Routledge \& Kegan Paul.

Leenders, Roger und Shaul Gabbay (Hrsg.). 1999. Corporate Social Capital and Liability. Norwell: Kluwer.

Lesser, Eric L. (Hrsg.). 2000. Knowledge and Social Capital. Foundations and Applications. Boston: Butterworth-Heinemann.

Lin, Nan. 1999. Social networks and status attainment. Annual Review of Sociology 25(1): 467-487.

Lin, Nan. 2001. Social Capital. A Theory of Social Structure and Action. New York: Cambridge University Press.

Lin, Nan und Yanjie Bian. 1991. Getting ahead in urban China. American Journal of Sociology 97(3): 657-688.

Lin, Nan, Karen Cook und Ronald S. Burt (Hrsg.). 2002. Social Capital. Theory and Research. New York: De Gruyter.

Loury, Glen C. 1977. A dynamic theory of racial income differences. S. 153-186 in Women, Minorities, and Employment Discrimination, hrsg. von Phyllis Wallace und Annette M. La Mond. Lexington: Heath.

Lüdemann, Christian und Sascha Peter. 2007. Kriminalität und Sozialkapital im Stadtteil. Eine Mehrebenenanalyse zu individuellen und sozialräumlichen Determinanten von Viktimisierungen. Zeitschrift für Soziologie 36(1): 25-42.

Luhmann, Niklas. 1969. Normen in soziologischer Perspektive. Soziale Welt 20(1): 28-48.

Luhmann, Niklas. 1980a. Talcott Parsons. Zur Zukunft eines Theorieprogramms. Zeitschrift für Soziologie 9(1): 5-17.

Luhmann, Niklas. 1980b. Gesellschaftsstruktur und Semantik. Studien zur Wissenssoziologie der modernen Gesellschaft. Band 1. Frankfurt a. M.: Suhrkamp.

Luhmann, Niklas. 1982. Liebe als Passion. Frankfurt a. M.: Suhrkamp.

Luhmann, Niklas. 1984. Soziale Systeme. Grundrißeiner allgemeinen Theorie. Frankfurt a. M.: Suhrkamp.

Mersch, Dieter und Michaela Ott (Hrsg.). 2007. Kunst und Wissenschaft. München: Fink.

Meyerson, Eva M. 2000. Human capital, social capital and compensation. The relative contribution of social contacts to managers' income. S. 287-311 in Knowledge and Social Capital. Foundations and Applications, hrsg. von Eric L. Lesser. Boston: Butterworth-Heinemann.

Nahapiet, Janine und Sumantra Ghoshal. 1998. Social capital, intellectual capital and the organizational advantage. Academy of Management Review 23(2): 242-266.

Paldam, Martin. 2000. Social capital: one or many? Definition and measurement. Journal of Economic Surveys 14(5): 629-653. 
Paldam, Martin und Gert T. Svendsen. 2000. An essay on social capital. Looking for the fire behind the smoke. European Journal of Political Economy 16(2): 339-366.

Parsons, Talcott. 1963. On the concept of influence. Public Opinion Quarterly 27(1): 27-62.

Parsons, Talcott. 1970 [1951]. The Social System. London: Free Press.

Parsons, Talcott. 1973 [1967]. Einige Grundzüge der allgemeinen Theorie des Handelns. S. 216-244 in Moderne amerikanische Soziologie, hrsg. von Heinz Hartmann. Stuttgart: Enke.

Parsons, Talcott. 1974. Religion in postindustrial America. The problem of secularization. Social Research 41(2): 193-225.

Parsons, Talcott. 1986. Aktor, Situation und normative Muster. Ein Essay zur Theorie sozialen Handelns. Frankfurt a. M.: Suhrkamp.

Paxton, Pamela. 1999. Is social capital declining in the United States? A multiple indicator Assessment. American Journal of Sociology 105(1): 88-127.

Portes, Alejandro. 1998. Social capital. Its origins and applications in modern sociology. Annual Review of Sociology 24(1): 1-24.

Powell, Walter W. und Laurel Smith-Doerr. 1994. Networks and economic life. S. 368-402 in Handbook of Economic Sociology, hrsg. von Neil J. Smelser und Richard Swedberg. Princeton: Princeton University Press.

Putnam, Robert D. 1993. The prosperous community. Social capital and public life. American Prospect 13(4): 35-42.

Putnam, Robert D. 1995. Bowling alone. America's declining social capital. The Journal of Democracy 6(1): 65-78.

Putnam, Robert D. 2000. Bowling Alone. New York: Simon \& Schuster.

Putnam, Robert D. (Hrsg.). 2001. Gesellschaft und Gemeinsinn. Sozialkapital im internationalen Vergleich. Gütersloh: Bertelsmann.

Reinbacher, Paul. 2008. Wissensdynamik in Communities. Sozialkapital und seine Wirkung auf die Lernfähigkeit sozialer Systeme. Wiesbaden: VS.

Schechler, Jürgen M. 2002. Sozialkapital und Netzwerkökonomik. Frankfurt a. M.: Peter Lang.

Scherke, Katharina. 2009. Emotionen als Forschungsgegenstand in der deutschsprachigen Soziologie. Wiesbaden: VS.

Schmid, A. Allan. 2000. Affinity as social capital. Its role in development. Journal of Socio-Economics 29(2): 159-171.

Schulz von Thun, Friedemann. 1981. Miteinander reden. Störungen und Klärungen. Psychologie der zwischenmenschlichen Kommunikation. Reinbek/H.: Rowohlt.

Schütz, Alfred. 1932. Der sinnhafte Aufbau der sozialen Welt. Eine Einleitung in die verstehende Soziologie. Wien: Springer.

Schütz, Alfred und Thomas Luckmann. 1975. Strukturen der Lebenswelt. Darmstadt: Luchterhand.

Simon, Fritz B. 2004. Zur Systemtheorie der Emotionen. Soziale Systeme 10(1): 111-139.

Spencer-Brown, George. 1969. Laws of Form. Portland: Cognizer.

Staubmann, Helmut. 1995. Die Kommunikation von Gefühlen. Ein Beitrag zur Soziologie der Ästhetik auf der Grundlage von Talcott Parsons' Allgemeiner Theorie des Handelns. Berlin: Duncker \& Humblot.

Staubmann, Helmut. 2004. Der affektive Aufbau der sozialen Welt. Soziale Systeme 10(1): 140-158.

Staubmann, Helmut. 2008. Ästhetik, Aisthetik, Emotionen. Soziologische Essays. Konstanz: UVK.

Szreter, Simon. 2000. Social capital, the economy, and education in historical perspective. S. 56-77 in Social Capital. Critical Perspectives, hrsg. von Stephen Baron, John Field und Tom Schuller. Oxford: Oxford University Press.

Tacke, Veronika. 2000. Netzwerk und Adresse. Soziale Systeme 6(2): 291-320. 
Tröndle, Martin und Julia Warmers (Hrsg.). 2012. Kunstforschung als ästhetische Wissenschaft. Beiträge zur transdiziplinären Hybridisierung von Wissenschaft und Kunst. Bielefeld: Transcript.

Veblen, Thorstein. 1973. The Theory of the Leisure Class. Boston: Houghton Mifflin.

Wasserman, Stanley und Katherine Faust. 1994. Social Network Analysis. Methods and Applications. Cambridge: Cambridge University Press.

Weber, Max. 1980 [1922]. Wirtschaft und Gesellschaft. Grundriss der verstehenden Soziologie. Tübingen: Mohr.

Weiss, Felix und Markus Klein. 2011. Soziale Netzwerke und Jobfindung von Hochschulabsolventen. Die Bedeutung des Netzwerktyps für monetäre Arbeitsmarkterträge und Ausbildungsadäquatheit. Zeitschrift für Soziologie 40(3): 228-245.

Wenger, Etienne C., Richard McDermott und William M. Snyder. 2002. Cultivating Communities of Practice. Boston: Harvard Business School Press.

Willke, Helmut. 1998. Systemisches Wissensmanagement. Stuttgart: Lucius \& Lucius.

Woolcock, Michael und Deepa Narayan. 2000. Social capital. Implications for development theory, research and policy. World Bank Research Observer 15(2): 225-250. 


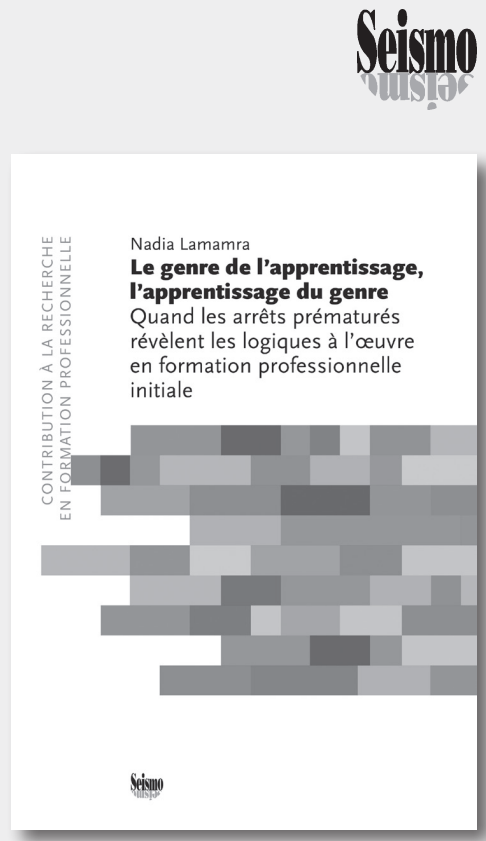

La formation professionnelle duale, au carrefour entre éducation et emploi, est représentative des tensions et enjeux propres à ces deux espaces. Cet ouvrage propose de la soumettre à une analyse de genre. Partant de la situation d'arrêts prématurés d'apprentissage, il questionne de manière plus large ce qui est produit et reproduit au cours de l'expérience apprentie. II interroge la façon dont la socialisation professionnelle participe d'une socialisation de genre. La formation professionnelle est ici analysée comme un lieu d'acculturation au travail, en particulier à l'un de ses piliers : la division sociale et sexuelle du travail. Cette grille de lecture donne l'occasion de réfléchir au statut des apprenti-e-s, à leur position particulière
Le genre de l'apprentissage, l'apprentissage du genre Quand les arrêts prématurés révèlent les logiques à l'œuvre en formation professionnelle initiale

296 pages, SFr. 38.-

dans l'organisation du travail. L'analyse de cette "fabrique du genre " permet d'expliquer le maintien des inégalités de sexe. Par ses questionnements, ce livre fait intervenir la sociologie de l'éducation et du travail, ainsi que les études genre et interroge, au-delà des seuls arrêts prématurés, des enjeux centraux de la formation professionnelle suisse.

Nadia Lamamra est docteure ès sciences sociales de l'Université de Lausanne, où elle a enseigné les études genre. Elle est responsable du champ de recherche "Processus d'intégration et d'exclusion » à l'Institut fédéral des hautes études en formation professionnelle (EHB IFFP IUFFP). Elle est membre du comité de rédaction de la revue Nouvelles Questions Féministes. 